\title{
PERCEPÇÕES DOS PROFISSIONAIS FARMACÊUTICOS QUANTO À IMPORTÂNCIA DAS DISCIPLINAS DA GRADE CURRICULAR DO CURSO DE FARMÁCIA
}

\author{
Nathália Ramos Ribeiro ROSA ${ }^{1}$, Gabriella Lima Viana CHAGAS ${ }^{1}$, Eduardo SHIMODA ${ }^{1 *}$, Fabrício Ferreira de \\ Albuquerque FERNANDES ${ }^{2}$, Alessandra Lobo da Silva ROSA ${ }^{1}$, Erik da Silva OLIVEIRA $^{1}$ \& Aldo SHIMOYA ${ }^{1}$ \\ 1 Universidade Candido Mendes. Campos dos Goytacazes, Rio de Janeiro, Brasil. \\ 2 Instituto Federal Fluminense - Campus Guarus. Campos dos Goytacazes, Rio de Janeiro, Brasil. \\ *Autor para correspondência: shimoda@ucam-campos.br \\ DOI: http://dx.doi.org/10.18571/acbm.171
}

\section{RESUMO}

No ano de 2002, o currículo de Farmácia passou por mudanças baseadas nas Diretrizes Curriculares editadas pelo Ministério de Educação, deixando de ter uma abordagem tecnicista, que dificultava a visão acadêmica e a sua capacidade crítica, para uma abordagem generalista. Considerando-se esta mudança de foco e as adequações curriculares envolvidas, o objetivo deste trabalho é identificar as disciplinas necessárias para que o farmacêutico realize suas funções durante o exercício profissional. Foi aplicado questionário a 30 farmacêuticos, no período de outubro a dezembro de 2015, para obtenção dos dados segundo as percepções dos profissionais quanto à importância de 65 disciplinas relacionados ao curso. Dentre as disciplinas avaliadas, podem ser citadas: "farmacologia clínica", "farmacotécnica", "análise orgânica", "anatomia humana", "homeopatia", dentre outras. Os farmacêuticos classificaram cada item como "não importante", "importante, mas não essencial", "essencial" ou "não sei", após o qual foi utilizado o método de Lawshe para validação de itens. Observou-se que, das 65 disciplinas contidas no questionário, 31 foram consideradas como essenciais ao exercício da profissão, sobressaindo-se a disciplina "farmacologia clínica" com 100\% dos entrevistados a considerando essencial. No caso de outras 28 disciplinas, não foi possível validá-las de forma definitiva como essenciais ou como a serem excluídas, em função do tamanho da amostra. Por outro lado, as disciplinas "farmacobotânica", "primeiros socorros", "bioestatística", "psicologia aplicada à farmácia", "fundamentos socioantropológicos de saúde" e "física aplicada à farmácia" poderiam ser excluídas da grade curricular do curso de farmácia. O trabalho poderá contribuir para uma análise crítica da grade curricular do curso e visando possíveis alterações e melhoria nesta.

Palavras chave: Farmácia; Grade curricular; Método de Lawshe; Questionário.

\section{ABSTRACT}

In 2002, the Pharmacy curriculum was revamped based on the curriculum guidelines issued by the Ministry of Education, while taking a technical approach, which hindered the academic view and its critical capacity, to a generalist approach. Considering this change in focus and curriculum adjustments involved, the objective of this study is to identify the necessary disciplines for the pharmacist to perform their duties during the professional practice. Questionnaire was applied to 30 pharmacists, from October to December 2015, to obtain the data in the perception of professionals about the importance of 65 subjects related to the course. Among the evaluated subjects can be cited: "clinical pharmacology," "pharmaceutical technology", "organic analysis," "human anatomy", "homeopathy", among others. Pharmacists rated each item as 


\section{ACTA

"not important", "important but not essential", "essential" or "do not know", after it was used Lawshe method for validating items. It was observed that, of the 65 subjects contained in the questionnaire, 31 were considered as essential to the profession, as the discipline "Clinical Pharmacology" 100\% of respondents considering essential. In the case of other 28 subjects it was not possible to definitively validating them as essential or as being deleted, depending on sample size. On the other hand, the subjects "pharmacobotany", "first aid", "biostatistics", "psychology applied to the pharmacy", "socioanthropological foundations of health" and "physics applied to pharmacy" could be excluded from the curriculum of the course of Pharmacy. The work will contribute to a critical analysis of the curriculum of the course and aiming for changes and improvement on this.

Keywords: Pharmacy; Curriculum; Lawshe method; Questionnaire.

\section{Introdução}

A formação profissional na área da saúde é influenciada diretamente pelos aspectos sóciopolíticos-econômicos além das tendências de mercados que norteiam as diferentes profissões. $\mathrm{O}$ Ministério da Saúde e Ministério da Educação vêm estimulando ações sociais pelos docentes da área da saúde, com o objetivo de conectar as necessidades da população brasileira aos conhecimentos técnico-científicos desenvolvidos por várias disciplinas ministradas nos cursos de graduação, reunindo valores que garantem a qualidade e emancipação dos futuros profissionais (ERDMANN et al., 2009).

O ensino superior em saúde, nas últimas décadas, tem sido alvo de profundas críticas. Em diversos países, tem crescido o questionamento sobre a capacidade dos cursos das Ciências da Saúde em cumprirem as finalidades gerais de seus programas, que deveriam desenvolver, no estudante, o potencial intelectual, a capacidade de análise, o julgamento e a avaliação crítica a habilidade para resolver problemas, o raciocínio crítico e a abordagem criativa (COSTA, 2009).

Dessa forma, os cursos de graduação precisam ter o sistema educacional revisado em relação à formação destes profissionais, por causa das diversas mudanças que tem acontecido como a crescente expansão tecnológica, o que muitas vezes se opõe aos sérios e complexos problemas de saúde enfrentados pela sociedade. (ERDMANN et al., 2009).

O farmacêutico deve ter a consciência da importância na atenção à saúde aos pacientes e sua responsabilidade neste processo, esta conscientização deve ter início no curso de graduação (PEREIRA, FREITAS, 2008). Além dessa conscientização sobre a sua responsabilidade nos processos de atenção à saúde, ele precisa estar dotado de conhecimentos, resultados da formação acadêmica e da vivencia profissional, para o efetivo exercício de suas atividades inerentes a assistência farmacêutica (OLIVEIRA et al., 2005). É necessário ao farmacêutico saber analisar e interpretar as prescrições de medicamentos, interações medicamento/medicamento e alimento/medicamento (SANTANA, 2013). O objetivo deste trabalho é identificar, por meio de um questionário, quais são as disciplinas necessárias para que o profissional farmacêutico realize suas funções durante o exercício da profissão.

\section{2 - Material e Métodos}

\subsection{Elaboração do Questionário}

Um questionário, de acordo com Chagas (2000), representa um conjunto de questões que gera dados necessários para alcançar os objetivos do projeto. Segundo Seufitelli (2011), a elaboração de 
questionários é a etapa de maior desafio e a mais interessante da pesquisa, pois o pesquisador deve entender todo o processo de raciocínio e comunicação do ser humano. O questionário é o processo de dados fornecidos pelo entrevistado, além de ser um critério usado como avaliador da pesquisa.

\subsection{Validação de Itens (Método de Lawshe)}

A qualidade de um meio de análise é elaborada por variáveis denominada por propriedades psicométricas, em que se encontra a confiabilidade e a validade. A confiabilidade é definida como o potencial do meio utilizado tem para avaliar fielmente um evento. Já a validade é o potencial do meio de análise usado tem para avaliar precisamente o evento considerado (PILATTI; PEDROSO; GUTIERREZ, 2010).

A validação de itens de questionário foi proposta por Lawshe (1975). Como já descrito por Dantier (2014) e Miranda (2014), o método se fundamenta na aplicação de questionários a especialistas que avaliam um item do questionário como (1) não importante; (2) importante, mas não essencial; (3) essencial; (N) não sei. Posteriormente, as respostas são agrupadas como essenciais ou não essenciais e, baseado em uma distribuição binomial, é verificada a validade do item, calculando uma taxa de validade de conteúdo em inglês CVR (Content validity Ratio), para cada um, conforme a fórmula a seguir:

$$
\mathrm{CVR}=\frac{\mathrm{n}_{\mathrm{e}}-(\mathrm{N} / 2)}{(\mathrm{N} / 2)}
$$

Onde:

- "ne" representa o número de especialistas que classificaram cada item como "essencial"

- "N" refere-se número total de respondentes, excluindo-se destes os que responderam "(N) Não sei/prefiro não opinar".

Lawshe (1975) define um valor mínimo de CVR, a partir da quantidade de especialistas que avaliam cada item para que estes sejam mantidos ou excluídos nas análises do questionário. Wilson, Pan e Schumsky (2012), estabeleceram uma nova tabela contendo valor de CVRcrítico (mínino) para cada item devido a anomalias encontradas na tabela de valores mínimos de CVR elaborada por Lawshe.

Por exemplo, de forma aleatória, para 20 respondentes, a probabilidade de que "Ne" especialistas assinalem a alternativa "essencial" pode ser calculada por análise combinatória, sendo apresentada na Tabela 1 a distribuição de probabilidades. 
Tabela 1: Distribuição de probabilidade binomial.

\begin{tabular}{ccccccccc}
\hline ENTREV. & $\mathbf{N}_{\mathbf{e}}$ & $\mathbf{N}_{\mathbf{n}}$ & $\begin{array}{c}\mathbf{P} \\
\text { (essencial) }\end{array}$ & $\begin{array}{c}\mathbf{P} \\
\text { (não) }\end{array}$ & Comb. & P(essencial) $^{\mathrm{n}}$ essencial & P(não) $^{\mathrm{n}_{\text {não }}}$ & P \\
\hline 20 & 0 & 20 & 0,5 & 0,5 & 1 & 1 & $9,53674.10^{-7}$ & 0,000001 \\
20 & 1 & 19 & 0,5 & 0,5 & 20 & 0,5 & $1,90735.10^{-6}$ & 0,000019 \\
20 & 2 & 18 & 0,5 & 0,5 & 190 & 0,25 & $3,8147.10^{-6}$ & 0,000181 \\
20 & 3 & 17 & 0,5 & 0,5 & 1140 & 0,125 & $7,62939.10^{-6}$ & 0,001087 \\
20 & 4 & 16 & 0,5 & 0,5 & 4845 & 0,0625 & $1,52588.10^{-5}$ & 0,004621 \\
20 & 5 & 15 & 0,5 & 0,5 & 15504 & 0,03125 & $3,05176.10^{-5}$ & 0,014786 \\
20 & 6 & 14 & 0,5 & 0,5 & 38760 & 0,015625 & $6,10352.10^{-5}$ & 0,036964 \\
20 & 7 & 13 & 0,5 & 0,5 & 77520 & 0,0078125 & 0,00012207 & 0,073929 \\
20 & 8 & 12 & 0,5 & 0,5 & 125970 & 0,00390625 & 0,000244141 & 0,120134 \\
20 & 9 & 11 & 0,5 & 0,5 & 167960 & 0,001953125 & 0,000488281 & 0,160179 \\
20 & 10 & 10 & 0,5 & 0,5 & 184756 & 0,000976563 & 0,000976563 & 0,176197 \\
20 & 11 & 9 & 0,5 & 0,5 & 167960 & 0,000488281 & 0,001953125 & 0,160179 \\
20 & 12 & 8 & 0,5 & 0,5 & 125970 & 0,000244141 & 0,00390625 & 0,120134 \\
20 & 13 & 7 & 0,5 & 0,5 & 77520 & 0,00012207 & 0,0078125 & 0,073929 \\
20 & 14 & 6 & 0,5 & 0,5 & 38760 & $6,10352.10^{-5}$ & 0,015625 & 0,036964 \\
20 & 15 & 5 & 0,5 & 0,5 & 15504 & $3,05176.10^{-5}$ & 0,03125 & 0,014786 \\
20 & 16 & 4 & 0,5 & 0,5 & 4845 & $1,52588.10^{-5}$ & 0,0625 & 0,004621 \\
20 & 17 & 3 & 0,5 & 0,5 & 1140 & $7,62939.10^{-6}$ & 0,125 & 0,001087 \\
20 & 18 & 2 & 0,5 & 0,5 & 190 & $3,8147.10^{-6}$ & 0,25 & 0,000181 \\
20 & 19 & 1 & 0,5 & 0,5 & 20 & $1,90735.10^{-6}$ & 0,5 & 0,000019 \\
20 & 20 & 0 & 0,5 & 0,5 & 1 & $9,53674.10^{-6}$ & 1 & 0,000001 \\
\hline
\end{tabular}

Logo após a distribuição binomial de probabilidades poderia ser feita a aproximação à distribuição normal que pode ser expressa pela de média e variância com as fórmulas:

- Média: $\mu=\mathbf{n} \cdot \mathbf{p}$

- Variância: $\sigma^{2}=\mathbf{n} \cdot \mathbf{p} .(1-\mathbf{p})$

Em que: "n" é o número de respondentes e "p" é a probabilidade de se assinalar "essencial" ( $p$ $=0,5)$. Com isso, para 20 respondentes, têm-se uma distribuição normal de média $=10$ e variância $=$ 5 (desvio-padrão = 2,236). Após isso, adota-se a significância de $5 \%$ na distribuição normal aproximada e obtém-se valor de $\mathrm{z}=1,96$ (Figura 1).

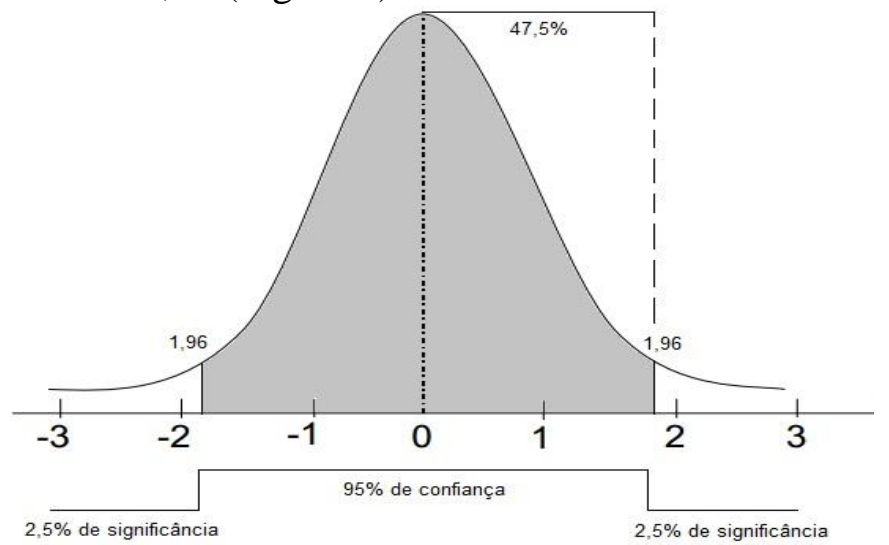

Figura 1: Distribuição normal e identificação do valor de z para 5\% de significância.

Levando em consideração que o valor crítico é igual a $\mu+$ z. $\sigma$, tem-se que o valor crítico é 10 $+1,96.2,236=14,4$, que corresponde ao mínimo de especialistas que precisa considerar o item como essencial para que ele seja válido. 
Lawshe (1975) propôs também o índice CVR, em que se a percentagem de especialistas que consideram um item essencial, que, em princípio, pode variar de $0 \%$ a 100\%, é transformada para valores de -1 a +1 (Figura 2).

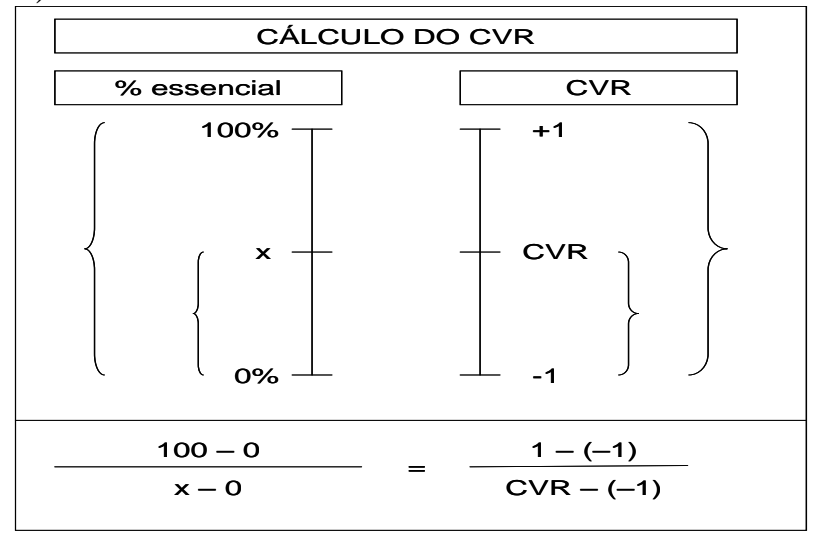

Figura 2: Interpolação para cálculo do CVR.

Depois disso, Wilson, Pan e Schumsky (2012), constataram inconsistência nos cálculos propostos por Lawshe e publicou um artigo novo corrigindo a tabela original. Ayre e Scally (2013) em seu estudo revisaram os métodos originais de cálculo do método de Lawshe, sugerindo métodos para cálculos iniciais de valores críticos e tabelas de probabilidades binomial exatas.

Diversos trabalhos na área médica aplicaram o método de Lawshe, podendo ser citados: fatores de risco associados à obesidade (GHAVAMZADEH; KHALKHALI; ALIZADEH, 2013), impactos psicossociais (ORELLANO; JUTAI, 2013), oncologia pediátrica (REEVE et al., 2013), qualidade da vida sexual feminina (MAASOUMI et al., 2013), autoconhecimento de pacientes com doença renal crônica (DEVRAJ; WALLACE, 2013).

A validação de itens foi usada também em outras áreas como escala de crença relacionada com a instrução assistida por computador (KOKLU, 2012), desempenho das empresas de construção chinesas (YAN, 2009), escala de habilidades de comunicação interpessoal (VAKILI; 2012), validação e confiabilidade de questionário sobre transporte coletivo urbano por ônibus em Campos dos Goytacazes, RJ (DANTIER, 2014), avaliação de itens de questionário, sob a percepção de clientes de uma churrascaria em Campos dos Goytacazes, RJ (MIRANDA, 2014), avaliação de um supermercado sob a percepção dos clientes: determinação da validade de itens do questionário (SALLES, 2015) e avaliação de pizzarias sob a percepção dos clientes: determinação da validade de itens do questionário (SOUZA, 2015).

\section{Metodologia}

O trabalho consistiu em um levantamento, por meio da aplicação de questionários aos farmacêuticos, entre os meses de outubro, novembro e dezembro de 2015, sendo as questões preenchidas diretamente pelos entrevistados. A elaboração do questionário consistiu nos seguintes passos:

Primeiro: Uma vez definido o questionário, foi feito um pré-teste. Este teste teve por objetivo avaliar o questionário antes da aplicação definitiva observando se os conteúdos abordados nas questões foram corretamente interpretados. Durante o teste piloto foi verificado que apenas um item do questionário deveria ser retirado e com isso feito a reformulação do questionário.

Segundo: Aplicação do questionário definitivo: cada farmacêutico demonstrou sua percepção quanto à importância das disciplinas do curso de graduação em Farmácia, que foram 65 itens, sendo 


\section{ACTA \\ Biomedica Brasiliensia}

oferecidas as seguintes opções de resposta para cada item: (1) não importante; (2) importante, mas não essencial; (3) essencial; (N) não sei. Foi obtida então, a frequência relativa (\%) de respondentes que consideravam o item essencial (FR essencial) através da razão entre os que assinalaram a opção " (3) essencial" e o total de entrevistados excluindo-se destes os que responderam "(N) não sei".

Esta FR essencial apresenta valores que podem variar de $0 \%$ a $100 \%$. Foi calculado, então, para cada item, o "Content Validity Ratio" (CVR) interpolando-se a FRessencial para valores de (-1) a $(+1)$.

Posteriormente, foi calculado o valor mínimo do CVR (CVR crítico) para que o item seja incluído no questionário. Como as respostas foram agrupadas como dicotômicas (essencial ou não essencial), estas seguem uma distribuição binomial, com possibilidade de aproximação para distribuição normal, com média $\mu=n$.p e variância $\sigma^{2}=n . p .(1-p)$ sendo $n=$ número de respondentes( excluindo-se os que assinalaram "não sei") e p= probabilidade de ocorrência como essencial, no qual adotou-se o valor igual a 0,5. Utilizando-se, o nível de significância de 5\%, foi calculado o CVR crítico.

No questionário havia perguntas referentes à área da farmácia que os farmacêuticos atuam, tempo de exercício de profissão e ano em que se formaram. Foram aplicados questionários a 30 farmacêuticos. As analises estatísticas foram processadas utilizando-se o aplicativo SAEG, versão 9.1, sendo apresentadas médias e erros- padrão, bem como gráficos de barra e de dispersão para análise dos resultados.

\section{Resultados e Discussão}

A Figura 3 apresenta as frequências relativas de respostas referentes ao grau de importância das disciplinas. Destaca-se na Figura 5 a disciplina farmacologia clínica, em que todos os entrevistados (100\%) a consideram uma disciplina essencial para realização do trabalho farmacêutico, ou seja, se mostra como a disciplina mais importante do curso superior de farmácia segundo os entrevistados, possivelmente isso se deve porque a farmacologia é uma das disciplinas mais usadas pelo profissional, podendo ser usada na indústria de medicamentos, em pesquisas de novos fármacos e no acompanhamento de pacientes em relação a conhecer os princípios ativos, orientar sobre reações adversas e possíveis interações entre medicamentos e com alimentos, ou seja, é a ciência que estuda os fármacos. 
ロ1- não importante

冈2-importante, mas não essencial

च3- essencial

Dão sei

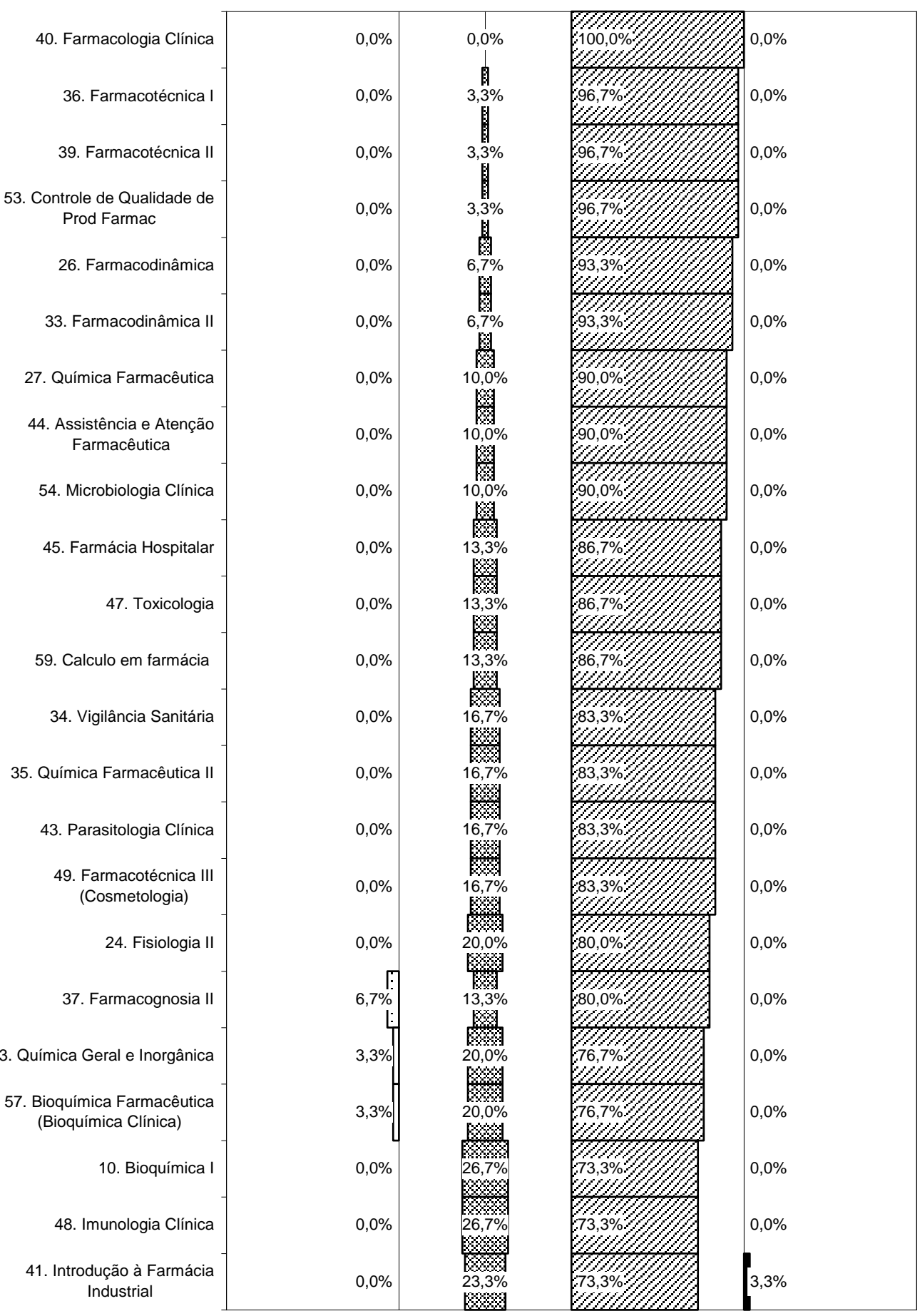

Figura 3: Frequência relativa (\%) de importância das disciplinas (Continua). 


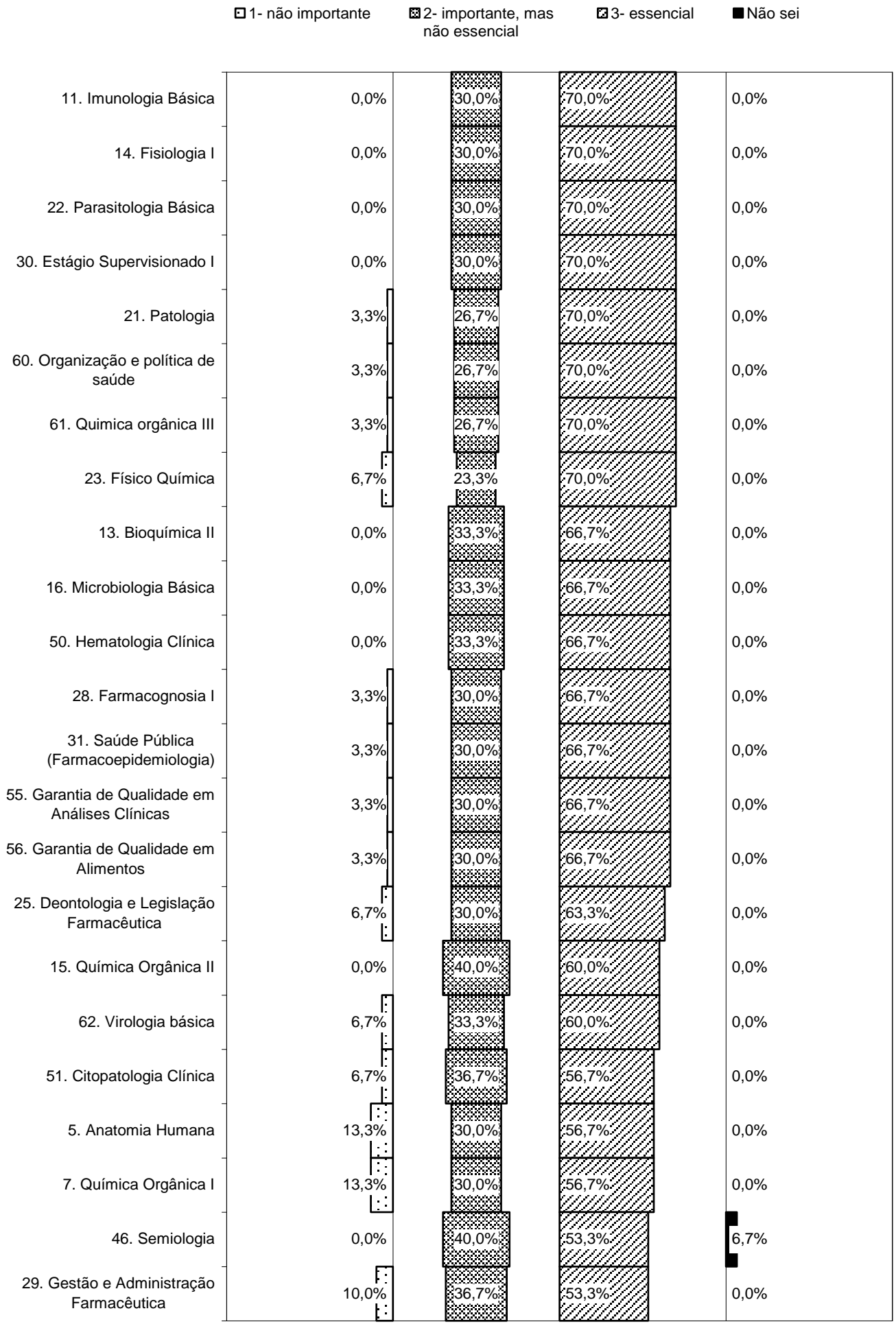

Figura 3: Frequência relativa (\%) de importância das disciplinas (Continua). 


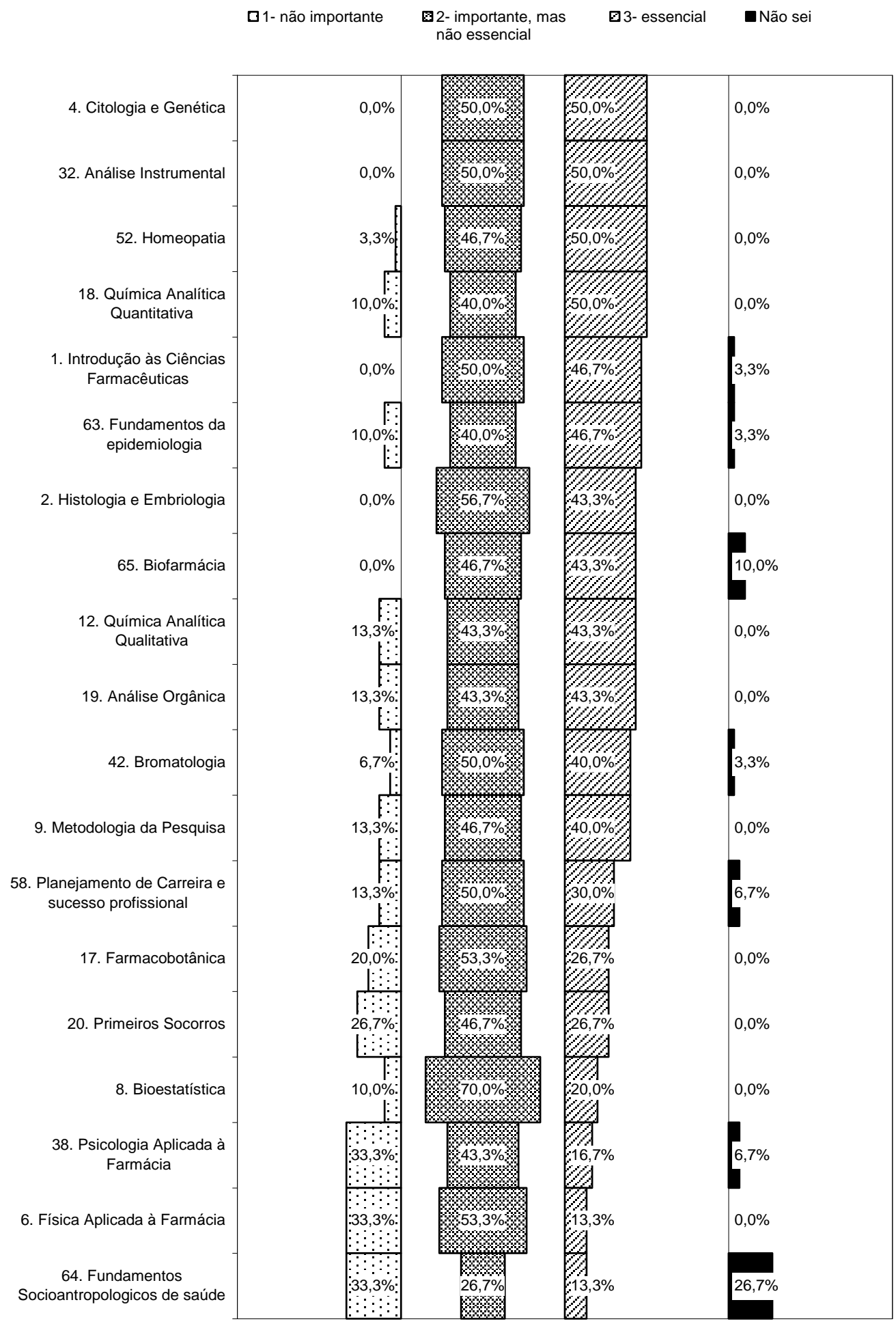

Figura 3: Frequência relativa (\%) de importância das disciplinas (Continuação).

Observa-se também que as disciplinas "Farmacotécnica I", "Farmacotécnica II" e "Controle de Qualidade de Produtos Farmacêuticos" apresentam a mesma avaliação, em que 96,7\% dos entrevistados os consideram essenciais e 3,3\% importante, mas não essencial e nenhum entrevistado as consideram disciplinas não importante. A disciplina "Fundamentos Sócio antropológicos de saúde" 
foi a que de todas obteve maior respostas de "não sei" com $26,7 \%$, isso pode ter ocorrido porque nem todas as faculdades contemplam essa disciplina na grade curricular, tornando-a desconhecida para a maioria dos farmacêuticos.

Analisando a Figura 4, podem-se verificar as médias e os erros-padrão no que diz respeito às questões relacionadas à importância dada pelos farmacêuticos aos itens avaliados. Observa-se que os itens com maiores valores são aqueles considerados mais relevantes na opinião dos farmacêuticos, ao passo que os itens com valores mais baixos possuem menor importância. Sendo assim, é possível perceber que as disciplinas consideradas mais importantes para os profissionais são: "Farmacologia Clínica" (3,0), "Farmacotécnica I" (2,97), "Farmacotécnica II" $(2,97)$, "Controle de Qualidade de Produtos Farmacêuticos" $(2,97)$, "Farmacodinâmica" $(2,93)$ e "Farmacodinâmica II" $(2,93)$. Isso se deve porque estas disciplinas são consideradas fundamentais e usadas com mais frequência no cotidiano do profissional. Enquanto que os itens considerados menos importantes para os profissionais foram: "Primeiros Socorros" $(2,0)$, "Fundamentos Socioantropologicos de saúde" $(1,83)$, "Psicologia Aplicada à Farmácia" $(1,82)$ e "Física Aplicada à Farmácia" $(1,80)$.

Com este resultado, observa-se a grande importância da maioria dos itens utilizados para avaliar a grade curricular do curso de farmácia. De 65 disciplinas avaliadas, 62 delas possuem uma média acima de 2, sendo que o mais importante foi obteve a média de 3,0, o que demonstra a grande importância do que se está avaliando.

Com uma média abaixo de 2 observa-se apenas 3 itens: "Fundamentos Socioantropologicos de saúde" $(1,83)$, "Psicologia Aplicada à Farmácia" $(1,82)$ e "Física Aplicada à Farmácia" $(1,80)$. 


\begin{tabular}{|c|c|c|}
\hline & Médias e e & \\
\hline & 1 & \\
\hline $\begin{array}{l}\text { 40. Farmacologia Clínica } \\
(n=30)\end{array}$ & & \\
\hline 36. Farmacotécnica I $(n=30)$ & 2 , & \\
\hline 39. Farmacotécnica II $(n=30)$ & 2 & \\
\hline $\begin{array}{l}\text { 53. Controle de Qualidade de } \\
\text { Prod Farmac }(n=30)\end{array}$ & 2 & \\
\hline 26. Farmacodinâmica $(n=30)$ & 2,9 & \\
\hline 33. Farmacodinâmica II $(n=30)$ & 2,5 & \\
\hline $\begin{array}{l}\text { 27. Química Farmacêutica } \\
\qquad(\mathrm{n}=30)\end{array}$ & 2,9 & \\
\hline $\begin{array}{l}\text { 44. Assistência e Atenção } \\
\text { Farmacêutica ( }(=30)\end{array}$ & 2,9 & \\
\hline $\begin{array}{l}\text { 54. Microbiologia Clínica } \\
\qquad(n=30)\end{array}$ & 2,9 & \\
\hline 45. Farmácia Hospitalar $(n=30)$ & 2,87 & \\
\hline 47. Toxicologia $(n=30)$ & 2,87 & \\
\hline $\begin{array}{l}\text { 59. Calculo em farmácia } \\
\qquad(n=30)\end{array}$ & 2,87 & \\
\hline 34. Vigilância Sanitária $(n=30)$ & 2,83 & \\
\hline $\begin{array}{l}\text { 35. Química Farmacêutica II } \\
\qquad(n=30)\end{array}$ & 2,83 & \\
\hline $\begin{array}{l}\text { 43. Parasitologia Clínica } \\
\qquad(n=30)\end{array}$ & 2,83 & \\
\hline $\begin{array}{l}\text { 49. Farmacotécnica III } \\
\text { (Cosmetologia) }(n=30)\end{array}$ & 2,83 & \\
\hline 24. Fisiologia II $(n=30)$ & 2,80 & $\longmapsto$ \\
\hline $\begin{array}{l}\text { 41. Introdução à Farmácia } \\
\text { Industrial }(n=30)\end{array}$ & 2,80 & \\
\hline $\begin{array}{l}\text { 3. Química Geral e Inorgânica } \\
\qquad(n=30)\end{array}$ & 2,73 & $\longmapsto$ \\
\hline 10. Bioquímica I $(n=30)$ & 2,73 & $\longrightarrow$ \\
\hline 37. Farmacognosia II $(n=30)$ & 2,73 & $\longrightarrow$ \\
\hline 48. Imunologia Clínica $(n=30)$ & 2,73 & $\longrightarrow$ \\
\hline $\begin{array}{l}\text { 57. Bioquímica Farmacêutica } \\
\text { (Bioquímica Clínica) }(n=30)\end{array}$ & 2,73 & $\longrightarrow$ \\
\hline
\end{tabular}

Figura 4: Média de importância das disciplinas (Continua). 


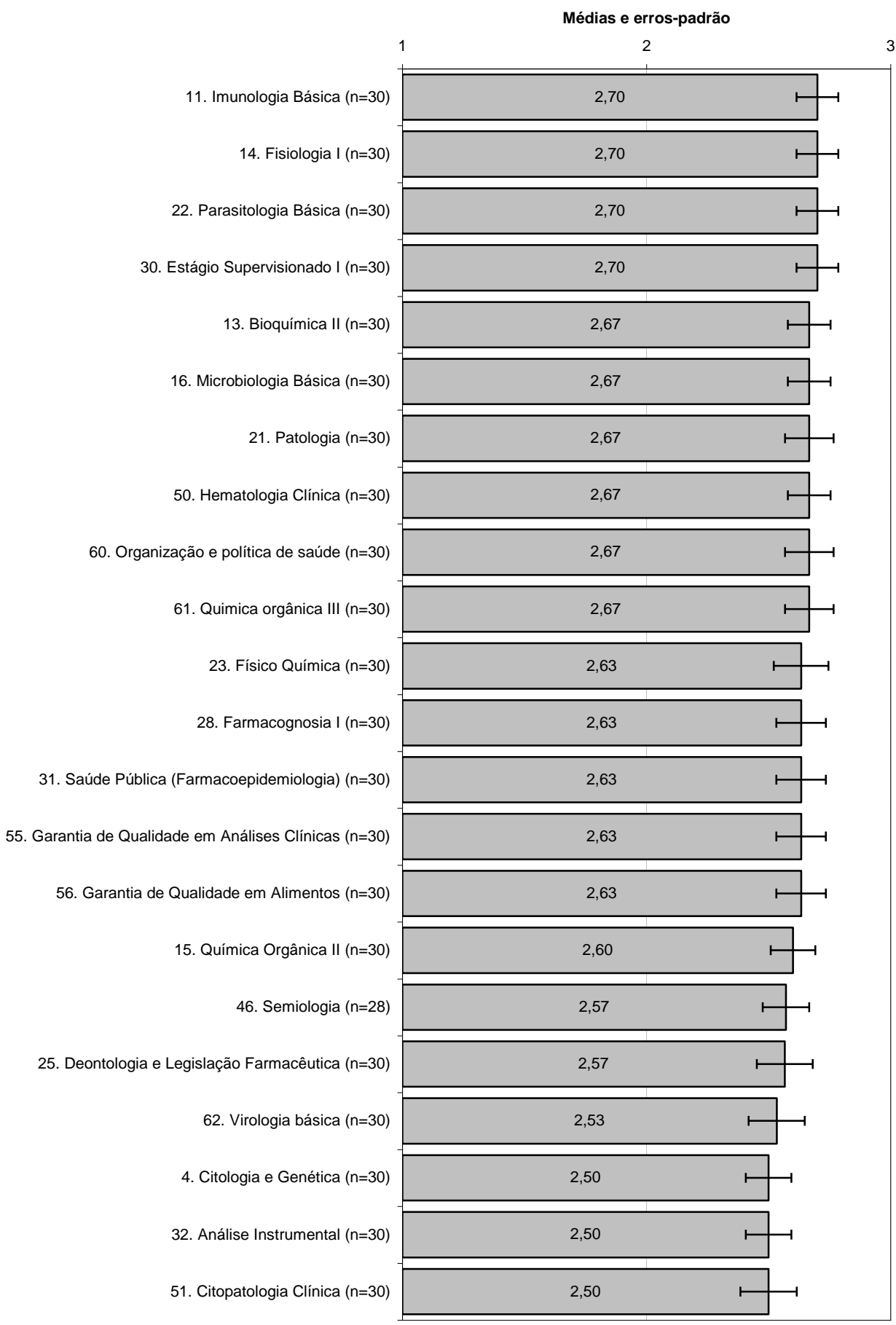

Figura 4: Média de importância das disciplinas (Continua). 


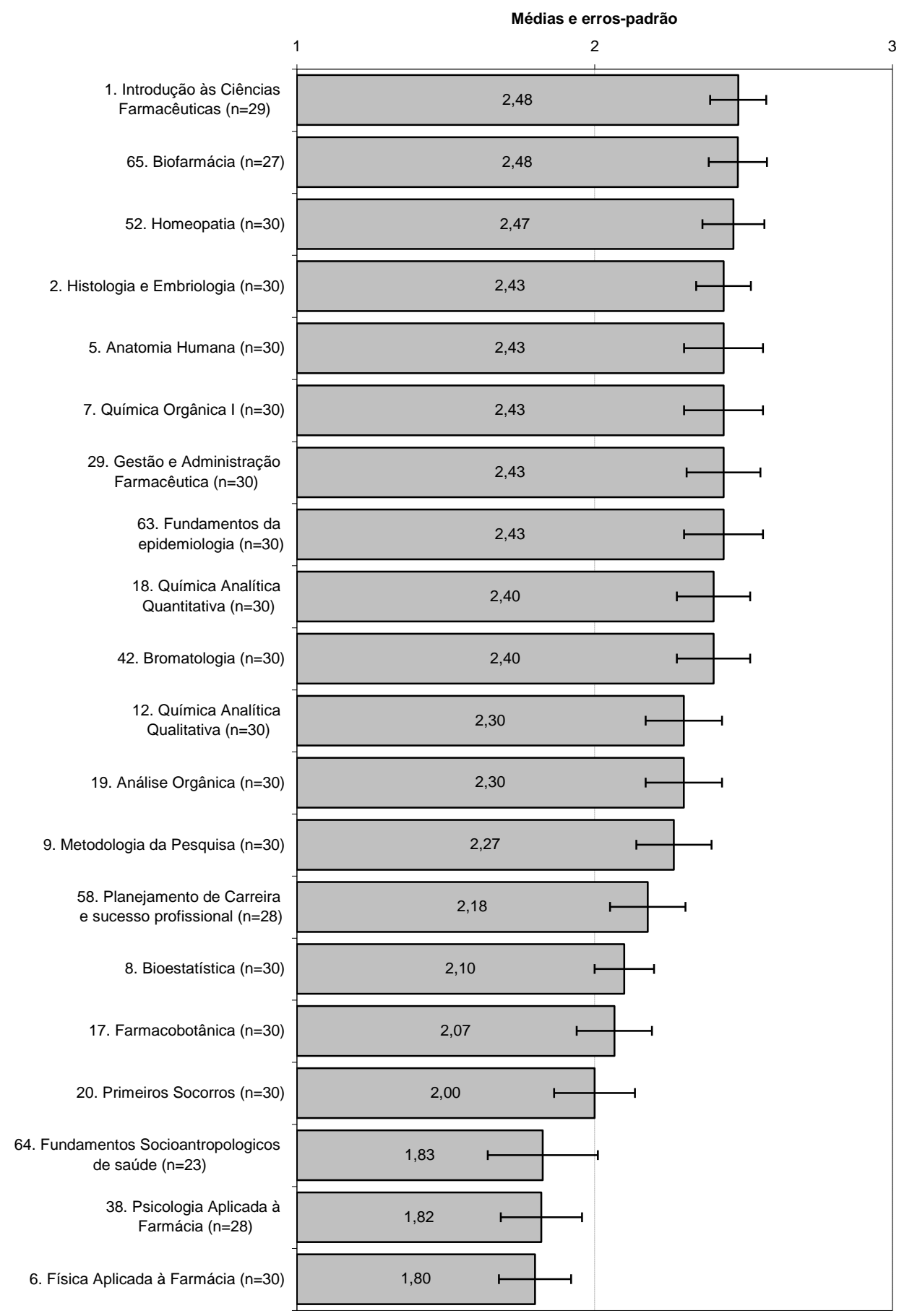

Figura 4: Média de importância das disciplinas (Continuação).

As Tabelas 2, 3 e 4 apresentam os cálculos do método de Lawshe, o número de farmacêuticos que considerou o item como essencial (Ne essencial), o número total de respondentes ( $\mathrm{Ne}$ ), excluindose os que responderam "(N) não sei", a percentagem que considerou o item como "essencial", os valores de CVR e CVRcrítico e a decisão.

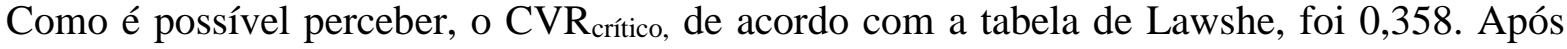
esse cálculo foram encontrados dos os CVRs referentes aos itens observados. Para todos os itens 
houve a comparação entre seus respectivos valores críticos com os o valor do CVR $=0,358$, isto é, para os valores acima de 0,358 os itens foram mantidos, os entre $-0,358$ e $+0,358$ estão no campo "reavaliar" e os abaixo de - 0,358 devem ser excluídos.

Observa-se na tabela 2 que os itens iniciais como "Farmacologia Clínica", "Farmacotécnica I", "Farmacotécnica II", "Controle de Qualidade de Produtos Farmacêuticos", "Farmacodinâmica", "Farmacodinâmica II", "Química Farmacêutica", "Assistência e Atenção Farmacêutica", "Microbiologia Clínica" e "Farmácia Hospitalar", por exemplo, estão denominados como disciplinas que devem ser mantidas na grade curricular do curso de farmácia, pois segundo os farmacêuticos entrevistados apresentam alta importância para o desenvolvimento das atividades farmacêuticas durante sua rotina de trabalho.

Tabela 2: Resultados do método de Lawshe quanto às disciplinas a serem mantidas, segundo a percepção de profissionais farmacêuticos.

\begin{tabular}{|c|c|c|c|c|c|c|}
\hline ITEM & $\mathrm{Ne}$ & $\mathbf{N}$ & $\% \mathrm{Ne}$ & CVR calc & CVRcrít & Decisão \\
\hline 40. Farmacologia Clínica & 30 & 30 & $100,0 \%$ & 1,000 & 0,358 & Manter \\
\hline 36. Farmacotécnica I & 29 & 30 & $96,7 \%$ & 0,933 & 0,358 & Manter \\
\hline 39. Farmacotécnica II & 29 & 30 & $96,7 \%$ & 0,933 & 0,358 & Manter \\
\hline 53. Controle de Qualidade de Prod Farmac & 29 & 30 & $96,7 \%$ & 0,933 & 0,358 & Manter \\
\hline 26. Farmacodinâmica & 28 & 30 & $93,3 \%$ & 0,867 & 0,358 & Manter \\
\hline 33. Farmacodinâmica II & 28 & 30 & $93,3 \%$ & 0,867 & 0,358 & Manter \\
\hline 27. Química Farmacêutica & 27 & 30 & $90,0 \%$ & 0,800 & 0,358 & Manter \\
\hline 44. Assistência e Atenção Farmacêutica & 27 & 30 & $90,0 \%$ & 0,800 & 0,358 & Manter \\
\hline 54. Microbiologia Clínica & 27 & 30 & $90,0 \%$ & 0,800 & 0,358 & Manter \\
\hline 45. Farmácia Hospitalar & 26 & 30 & $86,7 \%$ & 0,733 & 0,358 & Manter \\
\hline 47. Toxicologia & 26 & 30 & $86,7 \%$ & 0,733 & 0,358 & Manter \\
\hline 59. Calculo em farmácia & 26 & 30 & $86,7 \%$ & 0,733 & 0,358 & Manter \\
\hline 34. Vigilância Sanitária & 25 & 30 & $83,3 \%$ & 0,667 & 0,358 & Manter \\
\hline 35. Química Farmacêutica II & 25 & 30 & $83,3 \%$ & 0,667 & 0,358 & Manter \\
\hline 43. Parasitologia Clínica & 25 & 30 & $83,3 \%$ & 0,667 & 0,358 & Manter \\
\hline 49. Farmacotécnica III (Cosmetologia) & 25 & 30 & $83,3 \%$ & 0,667 & 0,358 & Manter \\
\hline 24. Fisiologia II & 24 & 30 & $80,0 \%$ & 0,600 & 0,358 & Manter \\
\hline 37. Farmacognosia II & 24 & 30 & $80,0 \%$ & 0,600 & 0,358 & Manter \\
\hline 3. Química Geral e Inorgânica & 23 & 30 & $76,7 \%$ & 0,533 & 0,358 & Manter \\
\hline 57. Bioquímica Farmac. (Bioquímica Clínica) & 23 & 30 & $76,7 \%$ & 0,533 & 0,358 & Manter \\
\hline 10. Bioquímica I & 22 & 30 & $73,3 \%$ & 0,467 & 0,358 & Manter \\
\hline 41. Introdução à Farmácia Industrial & 22 & 30 & $73,3 \%$ & 0,467 & 0,358 & Manter \\
\hline 48. Imunologia Clínica & 22 & 30 & $73,3 \%$ & 0,467 & 0,358 & Manter \\
\hline 11. Imunologia Básica & 21 & 30 & $70,0 \%$ & 0,400 & 0,358 & Manter \\
\hline 14. Fisiologia I & 21 & 30 & $70,0 \%$ & 0,400 & 0,358 & Manter \\
\hline 21. Patologia & 21 & 30 & $70,0 \%$ & 0,400 & 0,358 & Manter \\
\hline 22. Parasitologia Básica & 21 & 30 & $70,0 \%$ & 0,400 & 0,358 & Manter \\
\hline 23. Físico Química & 21 & 30 & $70,0 \%$ & 0,400 & 0,358 & Manter \\
\hline 30. Estágio Supervisionado I & 21 & 30 & $70,0 \%$ & 0,400 & 0,358 & Manter \\
\hline 60. Organização e política de saúde & 21 & 30 & $70,0 \%$ & 0,400 & 0,358 & Manter \\
\hline 61. Química orgânica III & 21 & 30 & $70,0 \%$ & 0,400 & 0,358 & Manter \\
\hline
\end{tabular}

Já na tabela 3, as disciplinas como "Bioquímica I", "Microbiologia Básica", "Semiologia", "Saúde Pública", "Hematologia Clínica" e outras estão no campo de "reavaliação", pois devem ser novamente analisadas quanto ao grau de importância e quanto à carga horária estabelecida. Semiologia farmacêutica refere-se à utilização dos métodos de semiologia adaptados à prática farmacêutica e voltados à prevenção e a condutas tomadas para problemas relacionados a medicamentos. Em relação a essa disciplina, o resultado foi inesperado, pois a semiologia está sendo 
muito tratada atualmente dentro da profissão farmacêutica.

Tabela 3: Resultados do método de Lawshe quanto às disciplinas a serem reavaliadas, segundo a percepção de profissionais farmacêuticos.

\begin{tabular}{|c|c|c|c|c|c|c|}
\hline ITEM & $\mathrm{Ne}$ & $\mathbf{N}$ & $\% \mathrm{Ne}$ & $\mathrm{CVR}_{\text {calc }}$ & CVR crít & Decisão \\
\hline 13. Bioquímica II & 20 & 30 & $66,7 \%$ & 0,333 & 0,358 & Reavaliar \\
\hline 16. Microbiologia Básica & 20 & 30 & $66,7 \%$ & 0,333 & 0,358 & Reavaliar \\
\hline 28. Farmacognosia I & 20 & 30 & $66,7 \%$ & 0,333 & 0,358 & Reavaliar \\
\hline 31. Saúde Pública (Farmacoepidemiologia) & 20 & 30 & $66,7 \%$ & 0,333 & 0,358 & Reavaliar \\
\hline 50. Hematologia Clínica & 20 & 30 & $66,7 \%$ & 0,333 & 0,358 & Reavaliar \\
\hline 55. Garantia de Qual. em Análises Clínicas & 20 & 30 & $66,7 \%$ & 0,333 & 0,358 & Reavaliar \\
\hline 56. Garantia de Qualidade em Alimentos & 20 & 30 & $66,7 \%$ & 0,333 & 0,358 & Reavaliar \\
\hline 25. Deontologia e Legislação Farmacêutica & 19 & 30 & $63,3 \%$ & 0,267 & 0,358 & Reavaliar \\
\hline 15. Química Orgânica II & 18 & 30 & $60,0 \%$ & 0,200 & 0,358 & Reavaliar \\
\hline 62. Virologia básica & 18 & 30 & $60,0 \%$ & 0,200 & 0,358 & Reavaliar \\
\hline 46. Semiologia & 16 & 28 & $57,1 \%$ & 0,143 & 0,370 & Reavaliar \\
\hline 5. Anatomia Humana & 17 & 30 & $56,7 \%$ & 0,133 & 0,358 & Reavaliar \\
\hline 7. Química Orgânica I & 17 & 30 & $56,7 \%$ & 0,133 & 0,358 & Reavaliar \\
\hline 51. Citopatologia Clínica & 17 & 30 & $56,7 \%$ & 0,133 & 0,358 & Reavaliar \\
\hline 29. Gestão e Administração Farmacêutica & 16 & 30 & $53,3 \%$ & 0,067 & 0,358 & Reavaliar \\
\hline 4. Citologia e Genética & 15 & 30 & $50,0 \%$ & 0,000 & 0,358 & Reavaliar \\
\hline 18. Química Analítica Quantitativa & 15 & 30 & $50,0 \%$ & 0,000 & 0,358 & Reavaliar \\
\hline 32. Análise Instrumental & 15 & 30 & $50,0 \%$ & 0,000 & 0,358 & Reavaliar \\
\hline 52. Homeopatia & 15 & 30 & $50,0 \%$ & 0,000 & 0,358 & Reavaliar \\
\hline 1. Introdução às Ciências Farmacêuticas & 14 & 29 & $48,3 \%$ & $-0,034$ & 0,364 & Reavaliar \\
\hline 65. Biofarmácia & 13 & 27 & $48,1 \%$ & $-0,037$ & 0,377 & Reavaliar \\
\hline 63. Fundamentos da epidemiologia & 14 & 30 & $46,7 \%$ & $-0,067$ & 0,358 & Reavaliar \\
\hline 2. Histologia e Embriologia & 13 & 30 & $43,3 \%$ & $-0,133$ & 0,358 & Reavaliar \\
\hline 12. Química Analítica Qualitativa & 13 & 30 & $43,3 \%$ & $-0,133$ & 0,358 & Reavaliar \\
\hline 19. Análise Orgânica & 13 & 30 & $43,3 \%$ & $-0,133$ & 0,358 & Reavaliar \\
\hline 9. Metodologia da Pesquisa & 12 & 30 & $40,0 \%$ & $-0,200$ & 0,358 & Reavaliar \\
\hline 42. Bromatologia & 12 & 30 & $40,0 \%$ & $-0,200$ & 0,358 & Reavaliar \\
\hline 58. Planej. de Carreira e sucesso profissional & 9 & 28 & $32,1 \%$ & $-0,357$ & 0,370 & Reavaliar \\
\hline
\end{tabular}

É possível observar também que 6 dos 65 itens se encontram na categoria de "excluir" do questionário, o que representa uma redução de $9,2 \%$ dos itens. Os itens que devem ser excluídos encontram-se na tabela 4 e são: "Farmacobotânica", "Primeiros Socorros", "Bioestatística", "Psicologia Aplicada à Farmácia", "Fundamentos Socioantropológicos de Saúde" e "Física Aplicada à Farmácia". Nenhum desses itens foi considerado essencial pela visão dos farmacêuticos.

Tabela 4: Resultados do método de Lawshe quanto às disciplinas a serem excluídas, segundo a percepção de profissionais farmacêuticos.

\begin{tabular}{lcccccc}
\hline ITEM & $\mathbf{N e}$ & $\mathbf{N}$ & $\mathbf{\%} \mathbf{N e}$ & $\mathbf{C V R}_{\text {calc }}$ & $\mathbf{C V R}_{\text {crít }}$ & Decisão \\
\hline 17. Farmacobotânica & 8 & 30 & $26,7 \%$ & $-0,467$ & 0,358 & Excluir \\
20. Primeiros Socorros & 8 & 30 & $26,7 \%$ & $-0,467$ & 0,358 & Excluir \\
8. Bioestatística & 6 & 30 & $20,0 \%$ & $-0,600$ & 0,358 & Excluir \\
38. Psicologia Aplicada à Farmácia & 5 & 28 & $17,9 \%$ & $-0,643$ & 0,370 & Excluir \\
64. Fundam. Socioantropológicos de Saúde & 4 & 23 & $17,4 \%$ & $-0,652$ & 0,409 & Excluir \\
6. Física Aplicada à Farmácia & 4 & 30 & $13,3 \%$ & $-0,733$ & 0,358 & Excluir \\
\hline
\end{tabular}

Um dos mais importantes campos de aplicação da pesquisa farmacobotânica é o da saúde em que uma grande porcentagem das matérias-primas empregadas pela indústria farmacêutica se compõe de espécies vegetais de todo tipo, embora os princípios ativos que constituem os medicamentos sejam 


\section{ACTA

às vezes produto de processos químicos. Essa disciplina não obteve tanta importância provavelmente porque pouco dos entrevistados trabalham na indústria farmacêutica, em que esta é mais empregada. Em relação a primeiros socorros pode ter sido porque quase nenhum dos profissionais realiza esse tipo de atividade no seu cotidiano, já psicologia aplicada à farmácia, ocorreu pelo fato dos profissionais acharem realmente desnecessário para carreira profissional do farmacêutico, o que é contraditório, pois é necessário que profissionais de saúde modifiquem sua maneira de olhar os processos de saúde-doença, para oferecer cuidados mais adequados aos indivíduos que atendem.

\section{Conclusões}

De acordo com os resultados obtidos, a grade curricular oferecida pelo curso de farmácia deve ser revista. Algumas disciplinas como "Farmacobotânica", "Primeiros Socorros", "Bioestatística", "Psicologia Aplicada à Farmácia", "Fundamentos Socioantropológicos de Saúde" e "Física Aplicada à Farmácia", de acordo com os resultados da pesquisa, não contribuem para o desenvolvimento das atividades farmacêuticas. Retirando essas disciplinas, poderia aumentar o tempo de outras disciplinas essenciais para o profissional como por exemplo "Farmacologia Clínica" e "Farmacotécnica", que de acordo com os resultados obtidos, são extremamente importantes para o farmacêutico e assim, capaz de melhor prepará-lo para o mercado de trabalho.

De todos 65 itens avaliados através da aplicação dos questionários aos farmacêuticos, alguns merecem destaque por apresentarem alta avaliação de importância, estes itens são: "Farmacologia Clínica" (100\%), "Farmacotécnica I" (96,7\%), “Farmacotécnica II” (96,7\%). É importante ressaltar que os itens avaliados com alta importância, são, portanto, os que inicialmente merecem maior atenção, pois necessitam de cargas horárias condizentes com o peso de sua importância na formação dos farmacêuticos. Muito embora a maioria dos itens esteja no campo "manter", existem também alguns itens que estão no campo "reavaliar", que não são considerados críticos, porém merecem também atenção, pois talvez poderia-se tomar medidas de melhor analisá-los e promover alterações quanto a carga horária ou em relação a didática da disciplina. Podem-se citar como exemplo as disciplinas "Bioquímica II", "Microbiologia Básica", "Farmacognosia I", "Saúde Pública (farmacoepidemiologia)" e "Hematologia Clínica".

A disciplina apontada como a mais crítica é a "Física Aplicada à Farmácia", em que 33,3\% dos entrevistados a considera não importante e 53,3\% importante, mas não essencial, sendo, portanto, o item que deve ser tratado imediatamente.

Sugere-se aos cursos de Farmácia, considerando que é recomendável a formação generalista inserir no mercado de trabalho, profissionais aptos a desenvolverem suas atividades farmacêuticas. Deste modo, pode-se explorar cada um dos resultados obtidos intervindo a fim de melhorar as deficiências identificadas, visando assim, melhorar os planos pedagógicos dos cursos de farmácia. A maior limitação deste estudo consistiu, principalmente, no pequeno tamanho da amostra (quanto maior o tamanho da amostra, mais representativo se torna o resultado).

\section{Referências}

AYRE, C.; SCALLY, A. J. Critical Values for Lawshe's Content Validity Ratio: Revisiting the Original Methods of Calculation. Measurement and Evaluation in Counseling and Development, v. 47, n. 1, p.79-86, 2013.

CHAGAS, Anivaldo Tadeu Roston. O Questionário na pesquisa científica. Fecap, Campinas, v. 1, n. 1, p.1-14, 2000. 
COSTA, Nilce Maria da Silva Campos. Formação pedagógica de professores de nutrição: uma omissão consentida? Revista de Nutrição, Campinas, v. 22, n. 1, p.97-104, 2009.

DANTIER, Rui Manuel Pinto et al. Validação e confiabilidade de questionário sobre transporte urbano coletivo em Campos dos Goytacazes, RJ. In: CONGRESSO LUSO-MOÇAMBICANO DE

ENGENHARIA / IV CONGRESSO DE ENGENHARIA DE MOÇAMBIQUE, 7., 2014, Inhambane/moçambique. Proceedings CLME2014 / IVCEM., 2014. p. 1 - 2.

DEVRAJ, Radhika; WALLACE, Lorraine S. Application of the content expert process to develop a clinically useful low-literacy Chronic Kidney Disease Self-Management Knowledge Tool (CKDSMKT). Research in Social and Administrative Pharmacy, v. 9, n. 5, p.633-639, 2013.

ERDMANN, Alacoque Lorenzini et al. O olhar dos estudantes sobre sua formação profissional para o Sistema Único de Saúde. Acta Paul. Enferm. São Paulo, v. 22, n. 3, p.288-294, 2009.

GHAVAMZADEH, Saeid; KHALKHALI, Hamid Reza; ALIZADEH, Mohammad. TV viewing, independent of physical activity and obesogenic foods, increases overweight and obesity. Journal of Health, Population and Nutrition, v. 31, n. 3, p.334-342, 2013.

KOKLU, Onder. Developing a belief scale related to computer assisted instruction. Energy Education Science and Technology Part B: Social and Educational Studies, v. 4, n. 3, p.17411752, 2012.

LAWSHE, C. H. A quantitative approach to content validity. Personnel Psychology, Nova Jersey, v. 28, n. 4, p.563-575, 1975.

MAASOUMI, Raziyeh et al. The sexual quality of life-female (SQOL-F) questionnaire: translation and psychometric properties of the Iranian version. Reproductive Health, Londres, v. 10, n. 1, p.16, 2013.

MIRANDA, Guilherme Melo. Avaliação de itens de questionário, sob a percepção de clientes de uma churrascaria em Campos dos Goytacazes, RJ. 2014. 46 f. Dissertação (Mestrado) - Curso de Engenharia de Produção, Universidade Candido Mendes, Campos dos Goytacazes, 2014.

OLIVEIRA, Andrezza B. et al. Obstáculos da atenção farmacêutica no Brasil. Revista Brasileira de Ciências Farmacêuticas, São Paulo, v. 41, n. 4, p.409-413, 2005.

ORELLANO, Elsa M.; JUTAI, Jeffrey W. Cross-cultural Adaptation of the Psychosocial Impact of Assistive Device Scale (PIADS) for Puerto Rican Assistive Technology Users. Assistive Technology, v. 25, n. 4, p.194-203, 2013.

PEREIRA, Leonardo Régis Leira; FREITAS, Osvaldo de. A evolução da Atenção Farmacêutica e a perspectiva para o Brasil. Revista Brasileira de Ciências Farmacêuticas, São Paulo, v. 44, n. 4, p.601-612, 2008. 
PILATTI, Luiz Alberto; PEDROSO, Bruno; GUTIERREZ, Gustavo Luis. Propriedades Psicométricas de Instrumentos de Avaliação: Um debate necessário. Revista Brasileira de Ensino de Ciência e Tecnologia, v. 3, n. 1, p.81-91, 2010.

REEVE, Bryce B. et al. The first step to integrating the child's voice in adverse event reporting in oncology trials: a content validation study among pediatric oncology clinicians. Pediatric Blood and Cancer, v. 60, n. 7, p.1231-1236, 2013.

SALLES, Sérgio Augusto Faria et al. Avaliação de um supermercado sob a percepção dos clientes: determinação da validade de itens do questionário. In: ENCONTRO INTERESTADUAL DE ENGENHARIA DE PRODUÇÃO, 1., 2015, São João da Barra. Anais Logística e Integração. Juiz de Fora: 2015. p. 1 - 12.

SANTANA, Priscila de Carvalho e Silva. Conhecimentos e habilidades necessários para o fornecimento de medicamentos para pacientes hospitalizados. Rio de Janeiro, 2013. Monografia (Residência Farmácia Hospitalar) - Faculdade de Farmácia, Universidade Federal Fluminense. Rio de Janeiro, 2013.

SEUFITELLI, Claudia Boechat. Identificação de pontos críticos em um curso superior de telecomunicações segundo percepção dos discentes. 2011. 71 f. Dissertação (Mestrado) - Curso de Mestrado em Pesquisa Operacional e Inteligência Computacional, Universidade Candido Mendes, Campos dos Goytacazes, 2011.

SOUZA, Juan Peçanha de et al. Avaliação de pizzarias sob a percepção dos clientes: determinação da validade de itens do questionário. Perspectivas Online, Campos dos Goytacazes, v. 13, n. 5, p.49-61, dez. 2015.

VAKILI, Mohammad Masoud; HIDARNIA, Ali Reza; NIKNAMI, Shamsaddin. Development and Psychometrics of an Interpersonal Communication Skills Scale (A.S.M.A) among Zanjan Health Volunteers. Journal of Hayat, v. 18, n. 1, p.5-19, 2012.

WILSON, F. R.; PAN, W.; SCHUMSKY, D. A. Recalculation of the Critical Values for Lawshe's Content Validity Ratio. Measurement and Evaluation in Counseling and Development, Londres, v. 45, n. 3, p.197-210, 2012.

YAN, Xiaoli. Establishment of Model of IT Performance Influence Factors for Chinese Construction Companies. International Conference on Management and Service Science, Wuhan, p.1-5, 2009. 